\title{
Protogenesis or Syngenesis? CL imaging and EBSD Orientation Mapping of Micro-inclusions in Yakutian diamonds
}

\author{
D.F. Wiggers de Vries ${ }^{1}$, D.A.M. de Winter $^{2}$, M.R. Drury ${ }^{3}$, G.R. Davies ${ }^{1}$, G.P. Bulanova ${ }^{4}$ \\ and D.G. Pearson ${ }^{5}$ \\ ${ }^{1}$ Faculty of Earth and Life Sciences, Vrije Universiteit Amsterdam, The Netherlands \\ ${ }^{2}$ Faculty of Sciences, Utrecht University, The Netherlands \\ ${ }^{3}$ Department of Earth Sciences, Utrecht University, The Netherlands \\ ${ }^{4}$ Department of Earth Sciences, University of Bristol, United Kingdom \\ ${ }^{5}$ Department of Earth Sciences, Durham University, United Kingdom
}

A new technique that combines electron backscattering diffraction (EBSD) orientation mapping with 3Dcathodoluminescence (3D-CL) is presented to study the origin of diamond inclusions. Many studies on diamonds focus on their inclusions and it is generally believed that the hosted minerals are syngenetic (Bulanova, 1995; Harris and Gurney, 1979; Meyer, 1985; Meyer, 1987; Mitchell and Giardini, 1953; Pearson and Shirey, 1999; Sobolev, 1977), as the inclusions often have epitaxial intergrowth with diamonds and an imposed diamond morphology. In addition, the relationship between $\mathrm{E}$ - and P-type paragenesis and the range in C-isotope composition of the host diamonds indicates that there is a genetic relation between mineral inclusions and the hosts. In contrast, Taylor et al. (2003) reported REE on Yakutian garnet inclusions that are not consistent with simultaneous growth and proposed a protogenetic origin for these inclusions.

In this study EBSD mapping is performed on a diamond plate surface and 3D-CL of a small area around an inclusion in the diamond to determine the orientation of the inclusion and the structure of the diamond growth zones near the inclusion. While previous studies of diamond inclusions mainly used Xray diffraction techniques to determine inclusion orientations, EBSD orientation mapping can qualitatively identify crystal directions. Also the structure of the diamond growth zones near the inclusions has never been investigated on the micron scale. Investigation of these zones is potentially a key tool to ascertain the inclusions origin: did they form simultaneously or earlier than the diamond?

\section{Methods}

The 3D-CL study focuses on a new technique (D.A.M. de Winter et al, in prep). This technique utilises sequential milling of micron slices with a focused ion beam (FIB) followed by CL imaging on each newly created surface. These consecutive CL images allow the nature of the diamond growth zones and the shape of the inclusion to be reconstructed in 3D.
Diamond plate 3615 from the Udachnaya pipe in Yakutia (Russia) was selected because of the presence of micro-inclusions and distinct growth patterns (see Fig. 1). Prior to milling EBSD mapping was performed on the polished $<110\rangle$ diamond plate incorporating the exposed inclusions. Slices were milled every 2 microns using a Ga ion beam at $30 \mathrm{kV}$ and $7 \mathrm{nA}$ on a FEI Nova Nanolab 600 (Utrecht University, The Netherlands).

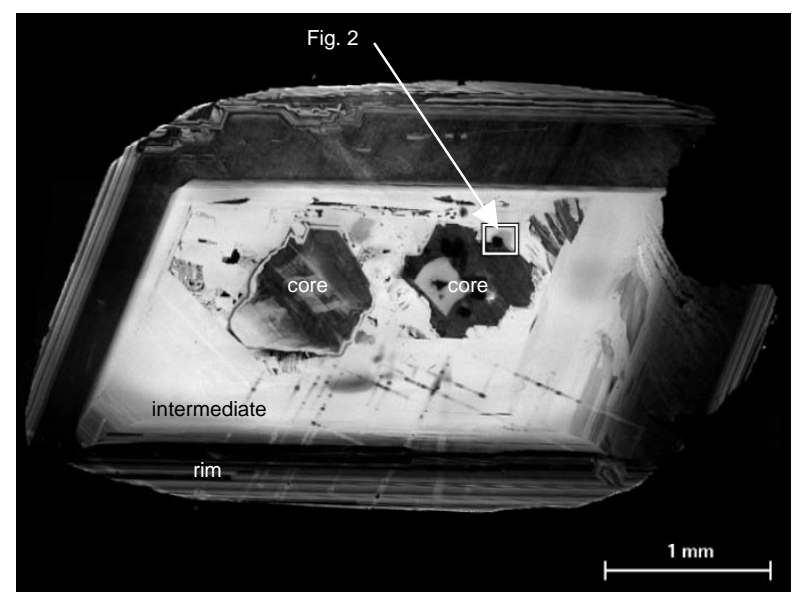

Fig. 1 CL image (SEM) of diamond 3615 (P-type), Yakutia. Two cubo-octahedral inside an elongated, octahedral crystal with a chromite inclusion located at the core-intermediate contact (see blow-up Fig. 2).

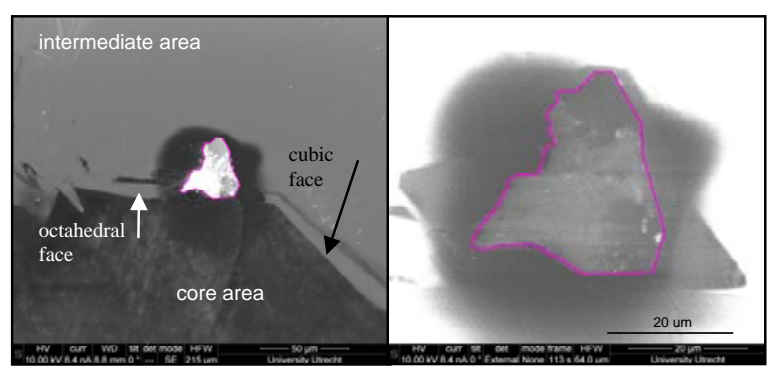

Fig. 2 Two mixed CL-SE images (FIB) of the exposed chromite inclusion (marked in pink) at the coreintermediate boundary with different magnification and black \& white contrast. The chromite is partly covered with conductive coating material. Around the chromite is a halo of diamond growth that locally obscures the CL characteristics of the core-rim contact. 


\section{Results}

EBSD mapping confirms that all exposed chromite inclusions and the diamond have cubic symmetry, and demonstrates that they all have the same orientation (matching within 0.4 degrees). The chromite selected for milling is shown in Fig. 2 and an overview of the created trench and milling direction are illustrated in Fig. 3. Around the chromite is a halo of diamond growth that locally obscures the CL characteristics of the core-rim contact. Fig. 5 \& 6 show some consecutive CL images of the milling progress. The outline of the chromite and the diamond growth halo are indicated in the different images and these data are combined to make a 3D-recontruction (see Fig. 4).

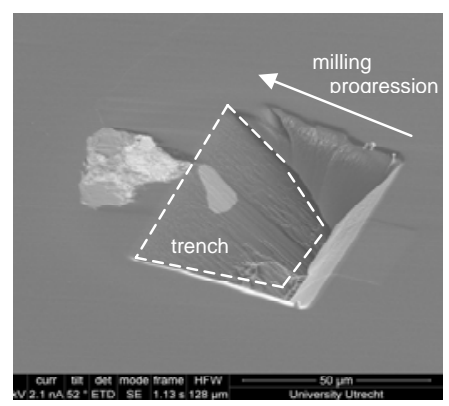

Fig. 3 Diamond plate surface $<110>$ with milled trench with inclined surfaces (outlined by dashed line) that exposes part of the inclusion at depth. The Milling progress is step-wise westward (Note figure is rotated $+90^{\circ}$ wrt Fig. 2).

\section{Discussion}

To help resolve a syngenesis-protogenesis origin of diamond inclusions, inclusion orientation and shape are important, as well as the nature of the diamond growth layers directly around the inclusion. In theory the geometry of mineral-host zones around proto- or syngenetic inclusions should be completely different. Diamond growth zones can grow around an inclusion while the inclusion has its own morphology (protogenetic), or the zones are intersected by the inclusion that has an imposed crystallography with many faces (syngenetic) (Bulanova, 1995).

A well formed face of the chromite inclusion is situated on the core-intermediate contact (see Fig. 6e) suggesting nucleation of the inclusion after formation of the diamond core. In Fig. 2, 5 \& 6 it is clear that there is a dark halo (no luminescence) of diamond growth surrounding the chromite. This halo is obscuring the very distinct core-intermediate contact. This generally weak CL signal could be caused by Fe or $\mathrm{N}$ impurities: Fe by suppressing $\mathrm{CL}$ and $\mathrm{N}$ by being absent (type-II diamond). While $\mathrm{N}$ is naturally occurring in the diamond lattice by replacement of a $\mathrm{C}$-atom and giving rise to CL, Fe could be present in the diamond due to diffusion from chromite. Both Fe and $\mathrm{N}$ were below the EDX detection limit $(<0.1 \mathrm{wt} \%)$, hence no quantitative results on these impurities were obtained.

The crystallographic shape of the halo (see Fig. 2) may suggest that it is a growth feature but the halo is also formed within the core suggesting a diffusive origin. In addition, the halo is not simply following the outline of the chromite, e.g. the halo is thicker in a region that has cubic faces (at position $\mathrm{X}$ in Fig. 4).

Diamond growth layers around the halo are complex. The fact that the growth layers in the rim of the halo (see Fig. 6e) grow around the chromite suggest a protogenetic origin for the chromite. In contrast, the imposed complex cubic morphology of the inclusion with its many faces indicates simulteaneous growth with the diamond. Perhaps speed or timing of growth is the determining factor, as protogenetic characteristics can appear when the chromite grew quicker than the diamond, or if both grew over distinct short time intervals that overlapped but that were not the same.

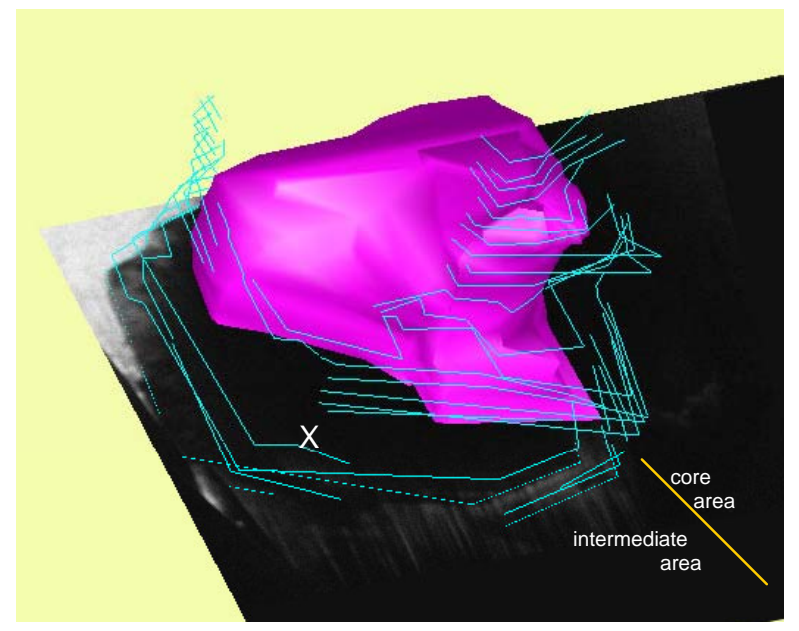

Fig. 4 3D-outline of the diamond halo (light blue lines) and the 3D-form of the chromite inclusion (in pink) at depth as exposed after milling. (Note figure is rotated $-90^{\circ}$ wrt Fig. 2).

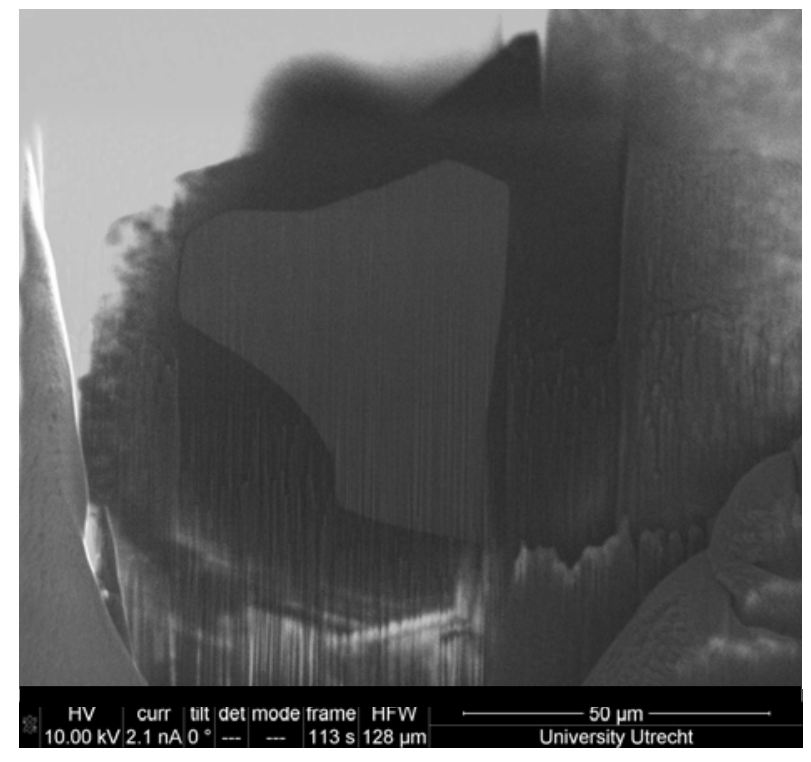

Fig. 5 CL image after last milling step exposing a section through the middle of the chromite. (Note figure is rotated $-90^{\circ}$ wrt Fig. 2). 


\section{Conclusion}

This work establishes that it is possible to obtain 3D$\mathrm{CL}$ at the micron-scale in diamonds. These initial data suggest that the chromite nucleated on the coreintermediate boundary and acted as a nucleation point for diamond growth. Both syngenetic and protogenetic criteria are present, and further study of the halo is necessary to resolve its origin.

\section{References}

Bulanova, G.P. (1995) The formation of diamond. Journal of Geochemical Exploration, 53(1-3), 1-23.

Harris, J.W. and Gurney, J.J. (1979) Inclusions in diamond. In J.E. Field, Ed. The properties of diamond, p. 555-591. Academic Press Inc. (London) LTD., London.

Meyer, H.O.A. (1985) Genesis of diamond: a mantle saga.
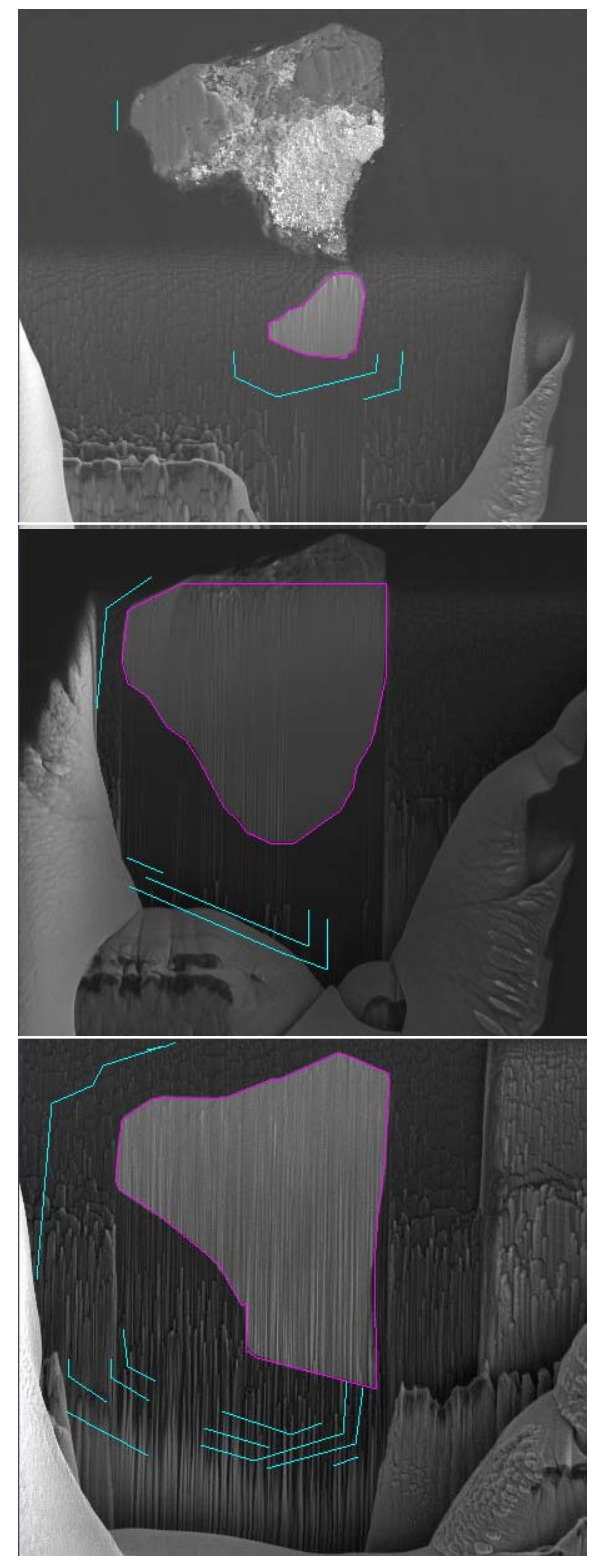

Fig. 6 Consecutive SE images (left-hand side) and CL images (right-hand side) of milling steps 10, 17 and 23 respectively (out of 23 steps total). The pink lines mark
American Mineralogist, 70(3-4), 344-355.

Meyer, H.O.A. (1987) Inclusions in diamond. In P.H. Nixon, Ed. Mantle Xenoliths, p. 501-523. John Wiley \& Sons Ltd.

Mitchell, R.S. and Giardini, A.A. (1953). Oriented olivine inclusions in diamond. American Mineralogist. 38, 136-138.

Pearson, D.G. and Shirey, S.B. (1999) Isotopic dating of diamonds. In D. Lambert, and J. Ruiz, Eds. Economic Geological Special Publications, SEG reviews, 12, p. 143-171.

Sobolev, N.V. (1977) Deep-seated inclusions in kimberlites and the problem of the composition of the upper mantle. 279 p. American Geophysical Union, Washington, D.C.

Winter, D.A.M. de, Lebbink, M.N., Wiggers de Vries, D.F. and Drury, M.R, (in prep)

Taylor, L.A., Anand, M., Promprated, P., Floss, C. and Sobolev, N.V. (2003) The significance of mineral inclusions in large diamonds from Yakutia, Russia. American Mineralogist, 88(5-6), 912-920.

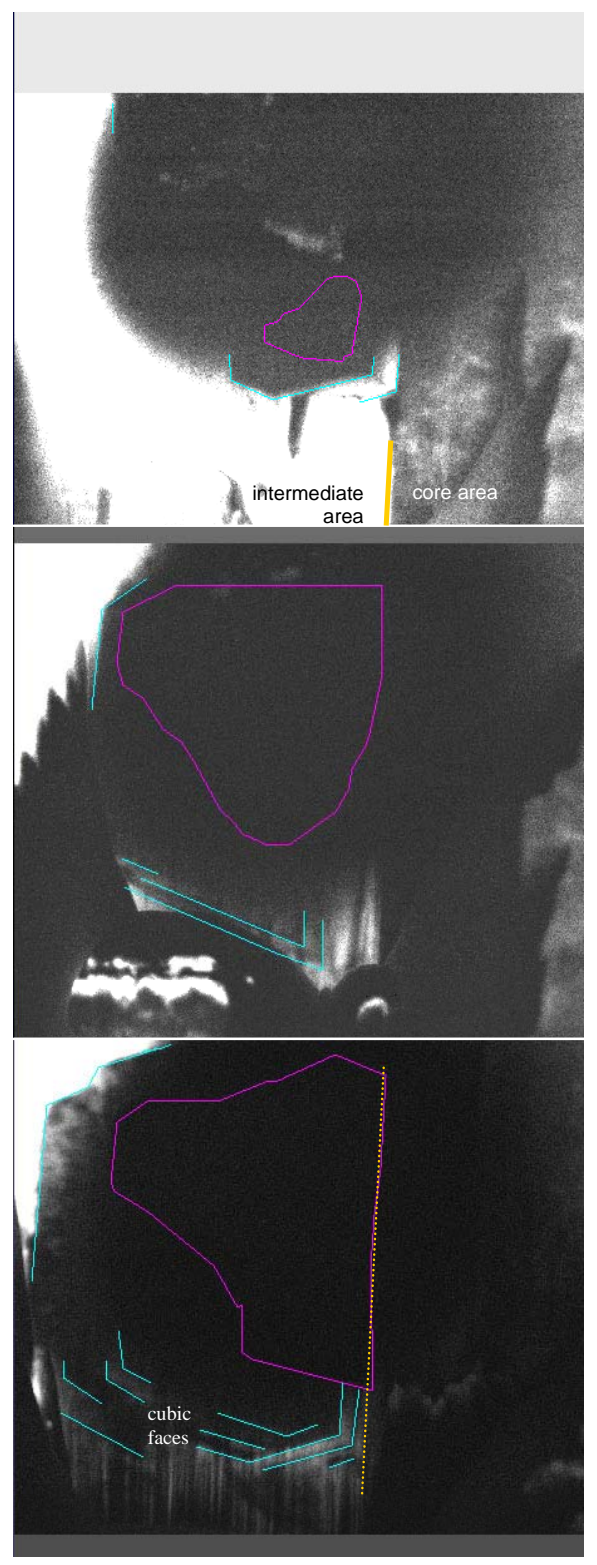

the outline of the chromite and the light blue lines the diamond growth layers within the halo. (Note figure is rotated $-90^{\circ}$ wrt Fig. 2). 